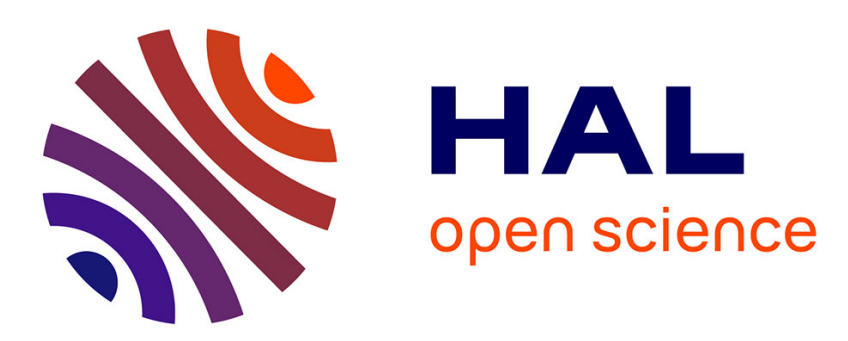

\title{
State Estimation Under Nonlinear State Inequality Constraints. A Tracking Application
}

Vincent Sircoulomb, Ghaleb Hoblos, Houcine Chafouk, José Ragot

\section{To cite this version:}

Vincent Sircoulomb, Ghaleb Hoblos, Houcine Chafouk, José Ragot. State Estimation Under Nonlinear State Inequality Constraints. A Tracking Application. 16th Mediterranean Conference on Control and Automation, MED'08, Jun 2008, Ajaccio, France. pp.1669-1674, 10.1109/MED.2008.4602024 . hal00292237

\section{HAL Id: hal-00292237 \\ https://hal.science/hal-00292237}

Submitted on 30 Jun 2008

HAL is a multi-disciplinary open access archive for the deposit and dissemination of scientific research documents, whether they are published or not. The documents may come from teaching and research institutions in France or abroad, or from public or private research centers.
L'archive ouverte pluridisciplinaire HAL, est destinée au dépôt et à la diffusion de documents scientifiques de niveau recherche, publiés ou non, émanant des établissements d'enseignement et de recherche français ou étrangers, des laboratoires publics ou privés. 


\title{
State Estimation Under Nonlinear State Inequality Constraints. A Tracking Application
}

\author{
Vincent Sircoulomb, Ghaleb Hoblos, Houcine Chafouk and José Ragot
}

\begin{abstract}
This paper proposes a general method for dealing with state estimation under nonlinear state soft inequality constraints. This method is based on the projection approach, and then has the advantage to be compatible with any kind of state estimator. In order to be taken into account, the nonlinear constraints are linearized about the constrained state using an iterated approach. The proposed algorithm is tested on a threedimension tracking application with nonlinear constraints on the moving body acceleration. The results are compared with those of an unconstrained Kalman filter.
\end{abstract}

\section{INTRODUCTION}

The Kalman filter is known to be the optimal estimator for linear systems corrupted by white state and measurement noises [1]. Its efficiency and ease of implementation have made this estimator be an essential tool for applications such as inertial navigation [2], target tracking [3], signal processing [4], optimal control [5] or fault diagnosis [6].

However, in the implementation of a Kalman filter, there is often known information about the process that is ignored because they do not fit easily in the structure of this estimator [7]. For example, when estimating a plane altitude, we know that the estimated value must be positive; when estimating a quaternion, its euclidian norm must be equal to one [8]. This kind of information can be interpreted as constraints on the state variables.

When the state variables are subject to linear equality constraints, different ways of dealing with this problem can be found. For example, it is possible to reduce the model parametrization [9], but this approach leads to a loss physical meaning associated to the state variables [7]. Another approach consists in treating the constraints as perfect measurements [10]; however, this method has the effect of leading to a singular measurement noise covariance matrix, which can cause numerical problems during the computation of the Kalman gain [7]. A third way of doing can be to use the Lagrange multiplier in order to take into account the constraints when deriving the Kalman filter from the optimality criterion chosen. The results obtained are different depending on the optimality criterion selected (least squares or maximum a posteriori [4]); however, this technique appears to be a particular case of the projection approach [7], [11]. This last unifying theory presents

\footnotetext{
This work was supported by the French arming delegation (DGA)

V. Sircoulomb, G. Hoblos and H. Chafouk are with the engineering school ESIGELEC, Technopôle du Madrillet, avenue Galilée, BP 10024, 76801 Saint-Étienne-du-Rouvray, France (sircoulomb, hoblos, chafouk) desigelec.fr

J. Ragot is with the CRAN (research laboratory on automatic control), Henry Poincaré University, 2 avenue de Haye, 54516 Vandœuvre-lès-Nancy, France jose.ragot@ensem. inpl-nancy. fr
}

undeniable advantages: It is physically meaningful, it can be used with any kind of state estimator and it is easily extendable to linear soft inequality constraints [12] via the active-set method [13]. Unfortunately, dealing with nonlinear state inequality constraints remains to be a delicate task. The purpose of this article is to propose a general method for dealing with nonlinear state soft inequality constraints. In this work, two contributions can be distinguished: The use of an iterated approach in order to deal with nonlinear state constraints via the projection approach, and the proposition of three different ways for associating a state estimator with some state constraints.

In this paper, the projection approach for estimating the state of a system under linear inequality constraints is reminded in section 2 . Then, section 3 proposes an iterated method for extending the projection approach to nonlinear state soft inequality constraints. The next section presents three different ways of coupling the projection approach with its associated state estimator. Finally, the results obtained by the proposed methods are compared in section 5 with those of an unconstrained Kalman filter on a 3D tracking application, with nonlinear constraints on the moving body acceleration.

\section{State ESTIMATION UNDER Linear STATE CONSTRAINTS: THE PROJECTION APPROACH}

Consider the following linear discrete time system:

$$
\left\{\begin{array}{l}
\boldsymbol{x}_{k+1}=\mathbf{A}_{k} \boldsymbol{x}_{k}+\mathbf{B}_{k} \boldsymbol{u}_{k}+\mathbf{G}_{k} \boldsymbol{w}_{k} \\
\boldsymbol{y}_{k}=\mathbf{C}_{k} \boldsymbol{x}_{k}+\boldsymbol{v}_{k}
\end{array}\right.
$$

where $\boldsymbol{x}_{k} \in \mathbb{R}^{n_{x}}$ is the state vector, $\boldsymbol{u}_{k} \in \mathbb{R}^{n_{u}}$ the input vector, $\boldsymbol{y}_{k} \in \mathbb{R}^{n_{y}}$ the measurement vector, $\boldsymbol{w}_{k} \in \mathbb{R}^{n_{w}}$ the process noise, $\boldsymbol{v}_{k} \in \mathbb{R}^{n_{y}}$ the measurement noise and $k \in \mathbb{N}$ the (discrete) time. $\boldsymbol{x}_{0}, \boldsymbol{w}_{k}$ and $\boldsymbol{v}_{k}$ are supposed to be gaussian, with respective covariance $\mathbf{P}_{0}, \mathbf{Q}_{k}$ and $\mathbf{R}_{k}$. Moreover, $\boldsymbol{w}_{k}$ and $\boldsymbol{v}_{k}$ are supposed to be mutually uncorrelated, and uncorrelated with $\boldsymbol{x}_{0} . \mathbf{A}_{k}, \mathbf{B}_{k}, \mathbf{C}_{k}$ and $\mathbf{G}_{k}$ are known matrices, potentially time varying. The system described by (1) is assumed to be observable.

\section{A. Linear state equality constraints}

Now, suppose that at each time step $k, \boldsymbol{x}_{k}$ is subject to the following linear equality constraint:

$$
\mathbf{D}_{k} \boldsymbol{x}_{k}=\boldsymbol{d}_{k}
$$

where $\boldsymbol{d}_{k} \in \mathbb{R}^{n_{d}}$ is a known vector and $\mathbf{D}_{k}$ a known matrix of appropriate dimension. $\boldsymbol{d}_{k}$ and $\mathbf{D}_{k}$ are possibly timevarying quantities. Furthermore, $\mathbf{D}_{k}$ is supposed to be a full 


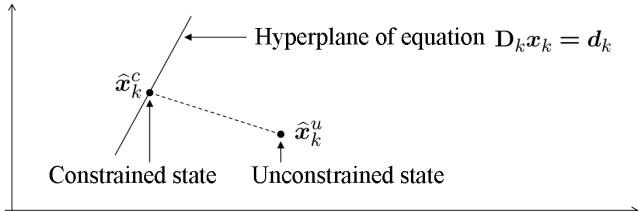

Fig. 1. Principle of the projection approach

rank matrice (if it is not the case, it means that there are some redundant constraints, which can be eliminated), and $n_{d}<n_{x}$.

We want to estimate the state $\boldsymbol{x}_{k}$ described by (1) and that this estimate satisfies (2). Let us denote by $\widehat{\boldsymbol{x}}_{k}^{u}$ the state estimated at time $k$ by an unconstrained estimator (i.e. an estimator which does not take into account the relation (2). This estimator can be for example a Kalman filter) and by $\widehat{\boldsymbol{x}}_{k}^{c}$ the estimate provided by same estimator, but which take into account equation (2). The associated covariances of estimation error are denoted $\mathbf{P}_{k}^{u}$ and $\mathbf{P}_{k}^{c}$. The principle of the projection approach is illustrated in Fig. 1. It consists in solving the following constrained optimization problem [7], [11]:

$$
\begin{aligned}
& \min _{\widehat{\boldsymbol{x}}_{k}^{c} \in \mathbb{R}^{n_{x}}}\left(\left(\widehat{\boldsymbol{x}}_{k}^{c}-\widehat{\boldsymbol{x}}_{k}^{u}\right)^{T} \mathbf{W}_{k}^{-1}\left(\widehat{\boldsymbol{x}}_{k}^{c}-\widehat{\boldsymbol{x}}_{k}^{u}\right)\right) \\
& \text { such that }: \mathbf{D}_{k} \widehat{\boldsymbol{x}}_{k}^{c}=\boldsymbol{d}_{k}
\end{aligned}
$$

where $T$ denotes he transposition operation and $\mathbf{W}_{k}$ is a symmetric positive definite weighting matrix. The solution is obtained through the use of the Lagrange multiplier, and summarized by the following set of equations [7], [11]:

$$
\begin{aligned}
\widehat{\boldsymbol{x}}_{k}^{c} & =\widehat{\boldsymbol{x}}_{k}^{u}+\mathbf{L}_{k}\left(\boldsymbol{d}_{k}-\mathbf{D}_{k} \widehat{\boldsymbol{x}}_{k}^{u}\right) \\
\mathbf{P}_{k}^{c} & =\left(\mathbf{I}_{n_{x}}-\mathbf{L}_{k} \mathbf{D}_{k}\right) \mathbf{P}_{k}^{u}\left(\mathbf{I}_{n_{x}}-\mathbf{L}_{k} \mathbf{D}_{k}\right)^{T} \\
\mathbf{L}_{k} & =\mathbf{W}_{k}^{-1} \mathbf{D}_{k}^{T}\left(\mathbf{D}_{k} \mathbf{W}_{k}^{-1} \mathbf{D}_{k}^{T}\right)^{-1}
\end{aligned}
$$

The constrained estimated state has the following properties [7], [11]:

- it is unbiased: $\forall k \in \mathbb{N}: \mathbb{E}\left(\boldsymbol{x}_{k}-\widehat{\boldsymbol{x}}_{k}^{c}\right)=\mathbf{0}_{n_{x} \times 1}$

- if $\mathbf{W}_{k}=\mathbf{P}_{k}^{u}$, then it results in the minimum variance filter: $\mathbb{E}\left(\left(\boldsymbol{x}_{k}-\widehat{\boldsymbol{x}}_{k}^{c}\right)\left(\boldsymbol{x}_{k}-\widehat{\boldsymbol{x}}_{k}^{c}\right)^{T}\right) \leq$ $\mathbb{E}\left(\left(\boldsymbol{x}_{k}-\boldsymbol{z}\right)\left(\boldsymbol{x}_{k}-\boldsymbol{z}\right)^{T}\right) \forall \boldsymbol{z} \in \mathbb{R}^{n_{x}}$

- if $\mathbf{W}_{k}=\mathbf{I}_{n_{x}}$, then it results in a constrained estimate that is closer to the true state than the unconstrained estimate: $\left\|\boldsymbol{x}_{k}-\widehat{\boldsymbol{x}}_{k}^{c}\right\| \leq\left\|\boldsymbol{x}_{k}-\widehat{\boldsymbol{x}}_{k}^{u}\right\|$.

where $\mathbf{I}_{\bullet}$ and $\mathbf{0}_{\bullet}$ are identity and zero matrices of appropriate dimension, and $\mathbb{E}$ is the mathematical expectation. It can also be seen thanks to (4) that if $\mathbf{W}_{k}=\mathbf{I}_{n_{x}}$, then the the unconstrained state is orthogonally projected onto the constraint hyperplan, which is generally not the case with $\mathbf{W}_{k}=\mathbf{P}_{k}^{u}$. Moreover, a parallel can be established between (4) and the equations for updating a Kalman filter. This can be explained by the fact that the constraint (2) can be interpreted as a fictive perfect measurement.

\section{B. Linear state soft inequality constraints}

Now, suppose that at each time step $k, \boldsymbol{x}_{k}$ is subject to the following linear soft inequality constraint:

$$
\mathbf{D}_{k} \boldsymbol{x}_{k} \leq \boldsymbol{d}_{k}
$$

where $\boldsymbol{d}_{k} \in \mathbb{R}^{n_{d}}\left(n_{d}<n_{x}\right)$ is a known time-varying vector and $\mathbf{D}_{k}$ a known full-rank time varying matrix of appropriate dimension. We want to estimate the state $\boldsymbol{x}_{k}$ described by (1) and that this estimate satisfies (5). A way of dealing with such a problem is using the active set method [13]. It consists in testing at each time step $k$ the $n_{d}$ scalar inequalities of (5). For the $i^{\text {th }}$ inequality $\left(1 \leq i \leq n_{d}\right.$ ), two scenarii can occur:

- The inequality is satisfied, and so do not have to be taken into account.

- The inequality is not satisfied (i.e. $\mathbf{D}_{i, k} \boldsymbol{x}_{k}>\boldsymbol{d}_{i, k}$ ). Then, an equality constraint is applied to the boundary: $\mathbf{D}_{i, k} \boldsymbol{x}_{k}=\boldsymbol{d}_{i, k}$, where for any matrix $\mathbf{M}_{k}$ at time $k$, $\mathbf{M}_{i, k}$ denotes the $i^{\text {th }}$ row of $\mathbf{M}_{k}$.

Consequently, dealing with soft inequality constraints reduces to the application at each time step of $n \leq n_{d}$ equality constraints. Such a problem has been treated in the first paragraph of this section.

\section{STATE ESTIMATION UNDER NONLINEAR STATE CONSTRAINTS}

Now, suppose that at each time step $k, x_{k}$ is subject to the following nonlinear equality constraint:

$$
\boldsymbol{g}_{k}\left(\boldsymbol{x}_{k}\right)=\boldsymbol{d}_{k}
$$

where $\boldsymbol{d}_{k} \in \mathbb{R}^{n_{d}}\left(n_{d}<n_{x}\right)$ is a known time-varying vector and $\boldsymbol{g}_{k}$ a known nonlinear function. We want to estimate the state $\boldsymbol{x}_{k}$ described by (1) and that this estimate satisfies (6). In this section, we only consider state equality constraints because we have seen in the previous section how to transform inequality constraints in equality constraints. As $\boldsymbol{g}_{k}$ is a nonlinear function, we cannot directly apply the projection method, because the Lagrange multiplier requires a linear constraint. A solution would be then to linearize $\boldsymbol{g}_{k}$ about the current constrained state estimate $\widehat{\boldsymbol{x}}_{k}^{c}$, through a first order Taylor development [7]. Let $\mathbf{D}_{k}$ be the following Jacobian:

$$
\mathbf{D}_{k}=\left.\frac{\partial \boldsymbol{g}_{k}}{\partial \mathbf{X}_{k}}\right|_{\mathbf{X}_{k}=\widehat{\boldsymbol{x}}_{k}^{c}}
$$

If the second and higher order terms are negligible, (6) becomes:

$$
\begin{array}{ll} 
& \boldsymbol{g}_{k}\left(\widehat{\boldsymbol{x}}_{k}^{c}\right)+\mathbf{D}_{k}\left(\boldsymbol{x}_{k}-\widehat{\boldsymbol{x}}_{k}^{c}\right)=\boldsymbol{d}_{k} \\
\Leftrightarrow \quad \mathbf{D}_{k} \boldsymbol{x}_{k}=\boldsymbol{d}_{k}-\boldsymbol{g}_{k}\left(\widehat{\boldsymbol{x}}_{k}^{c}\right)+\mathbf{D}_{k} \widehat{\boldsymbol{x}}_{k}^{c}
\end{array}
$$

which is indeed a linear constraint. Unfortunately, for linearizing (6) about $\widehat{\boldsymbol{x}}_{k}^{c}$, we need to know this state, and it can only be known by applying (4). We get here in a sticky problem, and we propose to use an iterated approach in order to solve it. The idea is the same than in the iterated extended Kalman filter [11], where the expansion of the nonlinear measurement equation is done several times, about 
a state that each time is theoretically closer to the a posteriori estimated state. This procedure is motivated by the parallel that can be established between the equations for applying a constraint (4) and those for updating the estimates of a Kalman filter [1]. The resulting algorithm is as follow:

1) Initialize with $\widehat{\boldsymbol{x}}_{k}^{(0)}=\widehat{\boldsymbol{x}}_{k}^{u}, \mathbf{P}_{k}^{(0)}=\mathbf{P}_{k}^{u}$, where $\widehat{\boldsymbol{x}}_{k}^{u}$ and $\mathbf{P}_{k}^{u}$ are computed by the unconstrained state estimator.

2) For $i=1, \ldots, N$, compute the following equations:

$$
\begin{aligned}
\mathbf{D}_{k}^{(i)} & =\left.\frac{\partial \boldsymbol{g}_{k}}{\partial \mathbf{X}_{k}}\right|_{\mathbf{X}_{k}=\widehat{\boldsymbol{x}}_{k}^{(i-1)}} \\
\boldsymbol{d}_{k}^{(i)} & =\boldsymbol{d}_{k}-\boldsymbol{g}_{k}\left(\widehat{\boldsymbol{x}}_{k}^{(i-1)}\right)+\mathbf{D}_{k}^{(i)} \widehat{\boldsymbol{x}}_{k}^{(i-1)} \\
\mathbf{L}_{k}^{(i)} & =\mathbf{W}_{k}^{-1} \mathbf{D}_{k}^{(i) T}\left(\mathbf{D}_{k}^{(i)} \mathbf{W}_{k}^{-1} \mathbf{D}_{k}^{(i) T}\right)^{-1} \\
\widehat{\boldsymbol{x}}_{k}^{(i)} & =\widehat{\boldsymbol{x}}_{k}^{u}+\mathbf{L}_{k}^{(i)}\left(\boldsymbol{d}_{k}^{(i)}-\mathbf{D}_{k}^{(i)} \widehat{\boldsymbol{x}}_{k}^{u}\right)
\end{aligned}
$$

3) Evaluate the constrained state estimate and associated covariance:

$$
\begin{aligned}
& \widehat{\boldsymbol{x}}_{k}^{c}=\widehat{\boldsymbol{x}}_{k}^{(N)} \\
& \mathbf{P}_{k}^{c}=\left(\mathbf{I}_{n_{x}}-\mathbf{L}_{k}^{(N)} \mathbf{D}_{k}^{(N)}\right) \mathbf{P}_{k}^{u} \\
& \times\left(\mathbf{I}_{n_{x}}-\mathbf{L}_{k}^{(N)} \mathbf{D}_{k}^{(N)}\right)^{T}
\end{aligned}
$$

\section{OPEN AND CLOSED LOOP CONSTRAINED FILTERS}

In this section, three ways of coupling an operator constraining some estimates ${ }^{1}$ through the projection approach with a stochastic state estimator (i.e. an estimator associating a covariance to its estimates) are presented. These three schemes, illustrated in Fig. 2, are:

- Open loop scheme,

- semi-closed loop scheme,

- closed loop scheme.

Originally, the state estimator and constraining operator are coupled in an open loop scheme [7]. But, at first sight, this scheme appears to be perfectible. Indeed, intuitively, it would seem to be a good idea to inform the recursive state estimator of the improvement achieved by the constraining operator ; this can be done by using the constrained state and covariance as entries of the recursive state estimator, as illustrated by the closed-loop scheme.

However, an advanced analysis shows that this procedure is extremely dangerous in the case of inequality constraints. Indeed, when an unconstrained state does not satisfy some inequality constraints, it is projected onto the surface of the constraint hypervolume, with respect to (3). Such a constrained state is then equivalent to an update issued from a fictive perfect measurement, as explained in section II.A. But the true state is into the constraint hypervolume, and not necessarily onto its surface, as illustrates Fig. 3. Then, the fictive measurement $\boldsymbol{g}_{k}\left(\boldsymbol{x}_{k}\right)=\boldsymbol{d}_{k}$ could no more be considered as a perfect one. Consequently, correcting the covariance with such a projected state is an illicit operation, leading to a decrease of the covariance. This can damage the filter robustness. This consideration leads us to the idea of semi-closed loop scheme.

\footnotetext{
${ }^{1}$ Subsequently, such an operator will be called state constrainer or constraining operator.
}

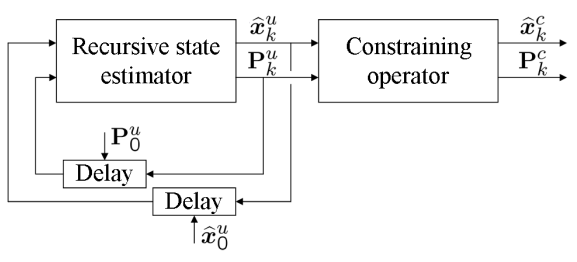

Open loop constrained state estimator

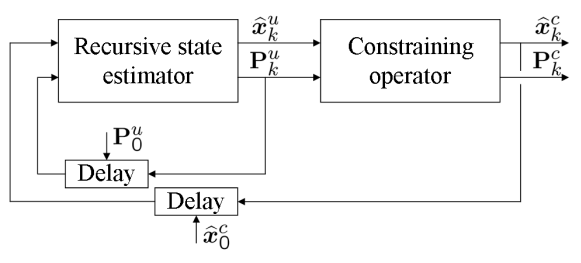

Semi-closed loop constrained state estimator

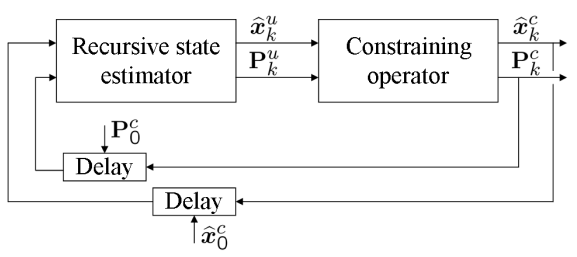

Closed loop constrained state estimator

Fig. 2. Open loop, semi-closed loop and closed loop constrained state estimator

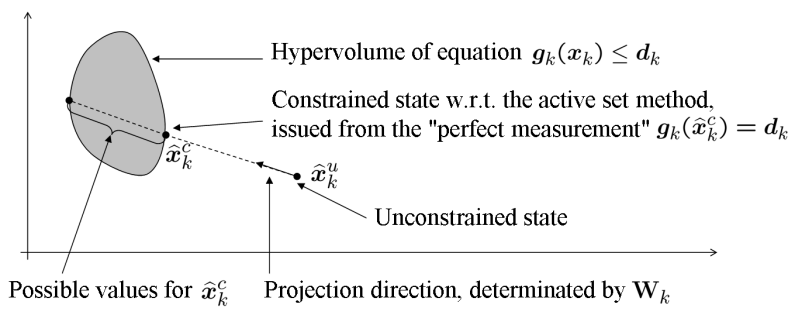

Fig. 3. The projection approach in the case of state inequality constraints

\section{Application}

In this section, we apply a constrained Kalman filter on a three-dimension tracking application, with nonlinear inequality constraints on the acceleration of the studied moving body. The results obtained are compared with those of an unconstrained Kalman filter. In such an application, the Kalman filter is one of the most powerful tools existing [14]. However, others approach exists, such as the Input Estimation (IE) in its original [15], recursive [16] and generalized [17] forms, the inclusion of the IE into a multiple hypothesis setting [18], or the Interacting Multiple Model [19] [20]. A complete state of the art can be found in [3].

\section{A. Model Considered}

Let $\vec{r}=\overrightarrow{O M}$ be the position vector of a moving body (a vehicle for example) in a three-dimension space. $O$ is fixed with regard to the earth, and $M$ is the body gravity centre. If we consider the earth as inertial (neither entrainment nor 
Coriolis acceleration), then we have:

$$
\vec{V}=\left.\frac{d \vec{r}}{d t}\right|_{[\mathcal{T}]} \quad \vec{\gamma}=\left.\frac{d \vec{V}}{d t}\right|_{[\mathcal{T}]}
$$

where $[\mathcal{T}]$ is an earth-fixed frame with origin $O, \vec{V}$ the body velocity (relatively to the earth), and $\vec{\gamma}$ the body acceleration (relatively to the earth). For the sake of convenience, the third axis of $[\mathcal{T}]$ is taken normal to the earth tangential plane at $O$. In this application, the body is supposed to have a hostile behaviour, i.e an unmeasured, varying and unpredictable acceleration. Then, we decide to model it as follow:

$$
\left.\frac{d \vec{\gamma}}{d t}\right|_{[\mathcal{T}]}=\vec{\eta}
$$

where $\vec{\eta}$ is a white noise. Projecting the equations (11) and (12) into $[\mathcal{T}]$ leads to the following model:

$$
\begin{aligned}
{\left[\begin{array}{c}
\dot{\boldsymbol{r}} \\
\dot{\boldsymbol{V}} \\
\dot{\boldsymbol{\gamma}}
\end{array}\right]=} & {\left[\begin{array}{ccc}
\mathbf{0}_{3 \times 3} & \mathbf{I}_{3} & \mathbf{0}_{3 \times 3} \\
\mathbf{0}_{3 \times 3} & \mathbf{0}_{3 \times 3} & \mathbf{I}_{3} \\
\mathbf{0}_{3 \times 3} & \mathbf{0}_{3 \times 3} & \mathbf{0}_{3 \times 3}
\end{array}\right]\left[\begin{array}{c}
\boldsymbol{r} \\
\boldsymbol{V} \\
\boldsymbol{\gamma}
\end{array}\right] } \\
& +\left[\begin{array}{c}
\mathbf{0}_{3 \times 1} \\
\mathbf{0}_{3 \times 1} \\
\mathbf{I}_{3}
\end{array}\right] \boldsymbol{\eta}
\end{aligned}
$$

where, for any vector $\vec{\lambda}, \boldsymbol{\lambda}$ is the projection of $\vec{\lambda}$ onto the axis of $[\mathcal{T}]$. This model is discretized thanks to an Euler scheme, with a sample time $T$ :

$$
\begin{aligned}
{\left[\begin{array}{l}
\boldsymbol{r}_{k+1} \\
\boldsymbol{V}_{k+1} \\
\boldsymbol{\gamma}_{k+1}
\end{array}\right]=} & {\left[\begin{array}{ccc}
\mathbf{I}_{3} & T \mathbf{I}_{3} & \mathbf{0}_{3 \times 3} \\
\mathbf{0}_{3 \times 3} & \mathbf{I}_{3} & T \mathbf{I}_{3} \\
\mathbf{0}_{3 \times 3} & \mathbf{0}_{3 \times 3} & \mathbf{I}_{3}
\end{array}\right]\left[\begin{array}{l}
\boldsymbol{r}_{k} \\
\boldsymbol{V}_{k} \\
\boldsymbol{\gamma}_{k}
\end{array}\right] } \\
& +\left[\begin{array}{c}
\mathbf{0}_{3 \times 3} \\
\mathbf{0}_{3 \times 3} \\
\mathbf{I}_{3}
\end{array}\right] \boldsymbol{w}_{k}
\end{aligned}
$$

where $\boldsymbol{w}_{k}=\int_{k T}^{(k+1) T} \eta(t) d t$ is a random walk [2], with covariance $\sqrt{T} \mathbb{E}\left(\eta \eta^{T}\right)$. From (14), one can easily identify $\boldsymbol{x}_{k}, \mathbf{A}_{k}$ and $\mathbf{G}_{k}$. The measurements $\boldsymbol{y}_{k}$ are done at each sample time thanks to a GPS which measure the body position. Through an appropriate coordinate change, we have:

$$
\boldsymbol{y}_{k}=\boldsymbol{r}_{k}+\boldsymbol{v}_{k}=\underbrace{\left[\begin{array}{lll}
\mathbf{I}_{3} & \mathbf{0}_{3 \times 3} & \mathbf{0}_{3 \times 3}
\end{array}\right]}_{=\mathbf{C}_{k}} \boldsymbol{x}_{k}+\boldsymbol{v}_{k}
$$

where $\boldsymbol{v}_{k}$ is the GPS measurement error, modelled by a white noise.

Moreover, from physical considerations, we have additional information about the body acceleration:

- The norm of the horizontal acceleration is below a bound $B_{h}$,

- the vertical acceleration is included in the interval $\left[B_{d} ; B_{u}\right]$.

By writing down $x_{k, i}$ the $i^{\text {th }}$ component of $\boldsymbol{x}_{k}$, and by seeing thanks to (14) that $\gamma_{k}=\left[\begin{array}{lll}x_{7, k} & x_{8, k} & x_{9, k}\end{array}\right]^{T}$, this
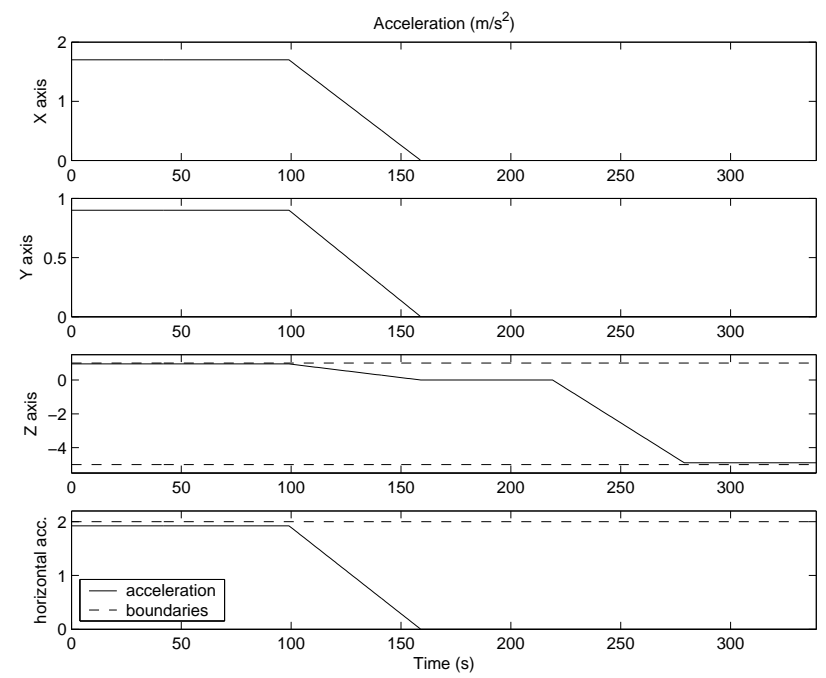

Fig. 4. Body true acceleration and state estimator boundaries

consideration leads to the following inequality constraints:

$$
\begin{gathered}
x_{7, k}^{2}+x_{8, k}^{2} \leq B_{h}^{2} \\
B_{d} \leq x_{9, k} \leq B_{u}
\end{gathered}
$$

\section{B. Experiments}

Equations (14) and (15) allow the use of a Kalman filter, while (16) requires the iterated method for taking itself into account. We decide to make the proposed Kalman filter works in SI units, and with sample time $T=1 \mathrm{~s}$.

The body initial condition is $\boldsymbol{x}_{0}=\left[\begin{array}{lll|l}15 & 20 & 0 & \ldots\end{array}\right.$. $\left.\begin{array}{llll|lll}\ldots & 0 & 0 & 0 & 1.7 & 0.9 & 0.95\end{array}\right]^{T}$, and the GPS standard-deviation is supposed to be $10 \mathrm{~m}$. The body acceleration is available in Fig. 4, where the horizontal acceleration is the euclidian norm of the $\mathrm{X}$ and $\mathrm{Y}$ axis acceleration.

The Kalman filter is tuned as follow: $\widehat{\boldsymbol{x}}_{0}=\mathbf{0}_{9 \times 1}, \mathbf{P}_{0}=$ $\operatorname{diag}\left(30^{2}, 30^{2}, 30^{2}, 30^{2}, 30^{2}, 30^{2}, 2^{2}, 2^{2}, 2^{2}\right), \quad \mathbf{Q}_{k}=0.2^{2} \mathbf{I}_{3}$ and $\mathbf{R}_{k}=10^{2} \mathbf{I}_{3}$, where "M $=\operatorname{diag}(\ldots)$ " is a shorthand notation indicating that $\mathbf{M}$ is a diagonal matrix fulfilled in order. The acceleration boundaries are set to the following values: $B_{h}=2 \mathrm{~m} / \mathrm{s}^{2}, B_{u}=1 \mathrm{~m} / \mathrm{s}^{2}, B_{d}=-5 \mathrm{~m} / \mathrm{s}^{2}$. By seeing Fig. 4, we can remark that the simulated acceleration is sometimes close to its boundaries, especially during the first hundred seconds. Then, we can expect that an unconstrained Kalman filter does violate (16).

Four kinds of Kalman filters are under consideration: One unconstrained and three constrained: One in open loop, one in semi-closed loop and one in closed loop. The number of iterations for applying the nonlinear state constraint (see section III.) is taken equal to $N=1$. The results obtained are presented in Fig. 5 to 8. For the sake of clarity, the estimated accelerations are separated in two figures (Fig. 5 and Fig. 6 ). We can clearly see that during the first hundred seconds and last sixty seconds, the unconstrained Kalman filter estimate goes over the limits determined by (16), whereas the open loop and semi-closed loop constrained filters do not. The closed loop constrained filter gives poor results for the horizontal acceleration estimates. The explanation is that the closed-loop scheme leads to a low covariance, and 

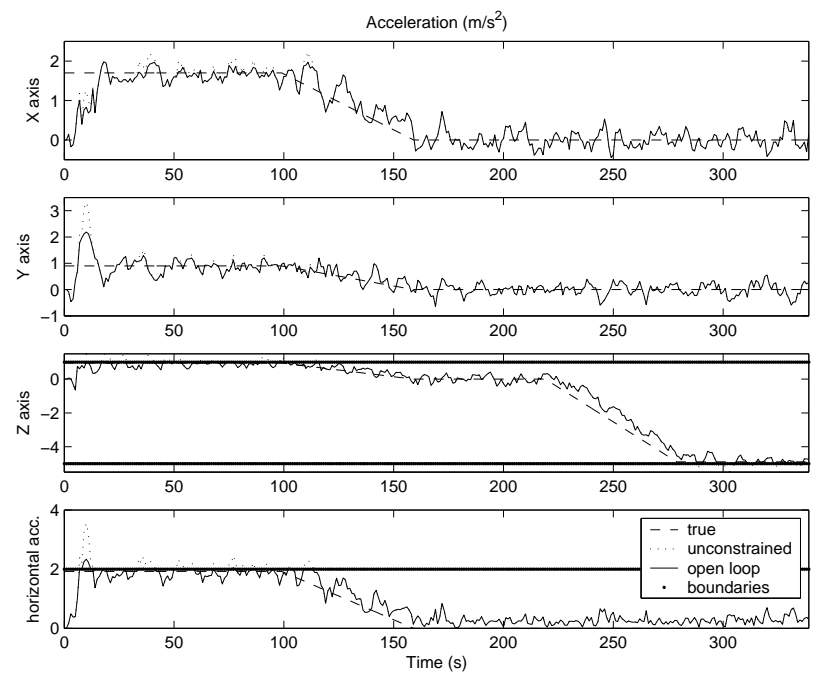

Fig. 5. True and estimated accelerations
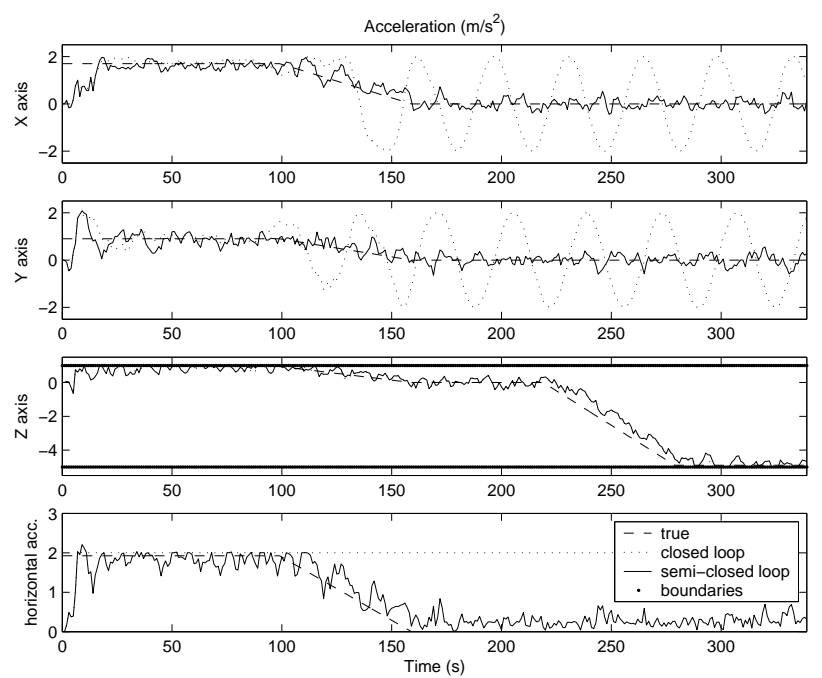

Fig. 6. True and estimated accelerations

consequently, a low Kalman gain. This is confirmed in Fig. 9, where the acceleration standard deviations (i.e. the square root of the corresponding diagonal terms of the covariances) of the closed loop constrained filter are clearly lower than the unconstrained filter ${ }^{2}$ ones. Then, as too few importance is given to observations, the closed loop constrained filter is unable to detect that after 100s, the nonlinear inequality of (16) is actually widely checked, and so, keep on applying it, as attests Fig. 6. This result confirms that the closed loop scheme is actually dangerous for state inequality constraints. The performance of the open loop and semi-closed loop constrained filters are equivalents and very satisfying. The position and velocitie estimation errors are shown in Fig. 7 and 8 .

In this experiment, the constrained filters were tuned with the value $N=1$ iteration. Now, let us see what happens if

${ }^{2}$ It should be reminded that, with respect to Fig. 2, the covariance propagated by the state estimator in the open loop and semi-closed loop schemes are the same than the unconstrained filter one.
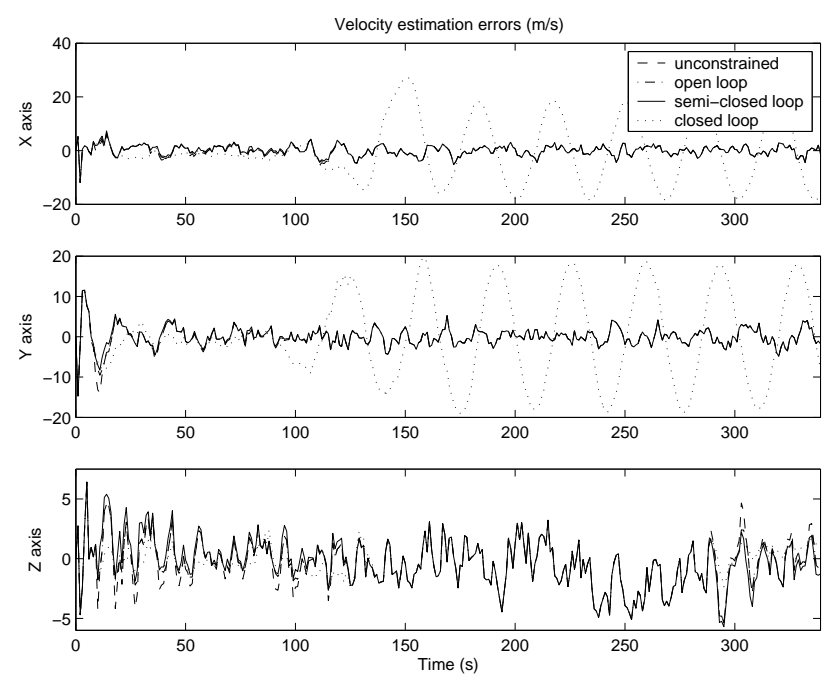

Fig. 7. Velocity estimation errors
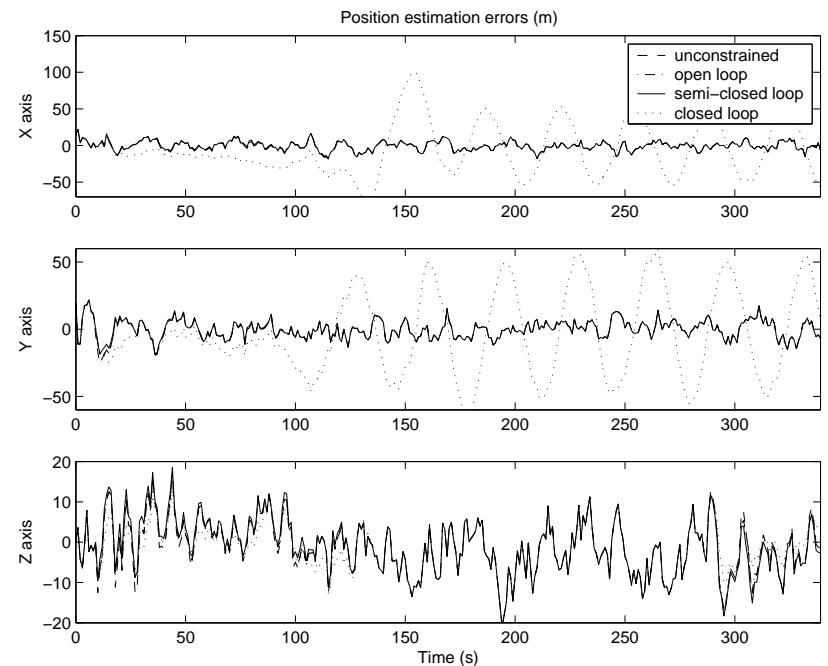

Fig. 8. Position estimation errors
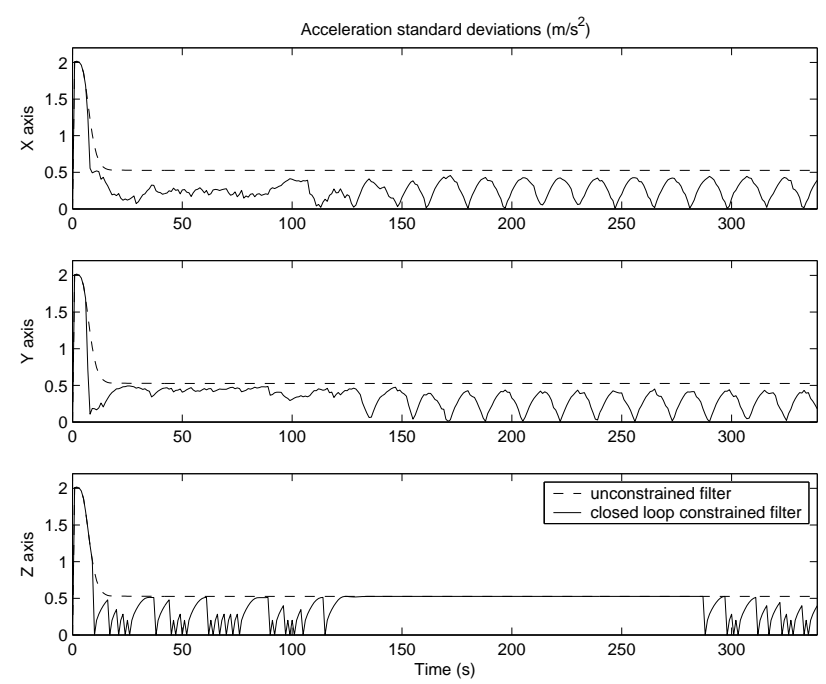

Fig. 9. Unconstrained filter, semi-closed loop constrained and closed loop constrained standard deviations 
TABLE I

ESTIMATION QUALITY IMPROVEMENT WITH RESPECT TO THE NUMBER OF ITERATIONS $N$

\begin{tabular}{|c||c|c|c|c|}
\hline$N$ & 1 & 2 & 5 & 20 \\
\hline Open loop scheme & $1.48 \%$ & $1.5 \%$ & $1.5 \%$ & $1.5 \%$ \\
\hline Semi-closed loop scheme & $2.07 \%$ & $2.09 \%$ & $2.09 \%$ & $2.09 \%$ \\
\hline
\end{tabular}

we change this value. As we could see that the closed loop constrained filter gives poor results, we do not consider it anymore. In order to evaluate the improvement of estimation quality of a constrained filter with a given $N$ relatively to an unconstrained filter $(N=0)$, we compute the following quantity $^{3}$ :

$$
\text { improvement }=100\left(1-\sqrt{\frac{\sum_{k=1}^{L}\left\|\boldsymbol{x}_{k}-\widehat{\boldsymbol{x}}_{k}^{c}\right\|^{2}}{\sum_{k=1}^{L}\left\|\boldsymbol{x}_{k}-\widehat{\boldsymbol{x}}_{k}^{u}\right\|^{2}}}\right)
$$

where $L$ is the simulation length, $\boldsymbol{x}_{k}$ is the true state at time $k$ and $\widehat{\boldsymbol{x}}_{k}^{c}$ the state estimated by the constrained filter considered, for a given $N$. The results, issued from an average of ten simulations, are presented in table I.

By seeing these results, we can conclude that $N=1$ iteration is a very good value for such an application. This result concurs with the fact that, for most problem treated with an iterated extended Kalman filter, the majority of the possible improvement is obtained by only relinearizing one time [11], p. 410. Moreover, the semi-closed loop scheme gives slightly better results than the open loop scheme, but the difference is not very obvious, as attest Fig. 5 and 6 . Finally, by seeing table I, the improvement provided by the constrained filters does not appears to be significant. This is because the body acceleration can be easily estimated, as the position is frequently measured: The acceleration estimated by an unconstrained Kalman filter does not often go over the boundaries, which explained the low improvement bringed.

\section{CONCLUSION}

In this paper, a way of dealing with state estimation under nonlinear soft inequality state constraints has been presented. This method is an extension of the projection approach using an iterated approach. Three ways of coupling the state constraining operator with the recursive state estimator has been exposed: open loop, semi-closed loop and closed loop. In the case of state inequality constraints, this last scheme is unsatisfactory because it leads to a severe covariance decrease, misleading the state estimator. The open loop and semi-closed loop schemes give equivalent and very satisfactory results. However, the semi-closed loop scheme could be preferred because it allows a reduction of the frequency application of the state constraining operator. A three-dimension tracking application highlighted these results, and allows us to see that one iteration for applying the state constraints is enough. In this application, the improvement provided by the consideration of constraints is not significant. However, it does not take away from

\footnotetext{
${ }^{3}$ This quantity computes the RMS (Root Mean Square) reduction in percent of the considered constrained filter estimation error in comparison to the unconstrained filter one.
}

the interest of the proposed approach in any way, because its general formulation make it is suitable for any kind of nonlinear constraints. For exemple, as potential application, we can mention the accelerometers bias and gyroscopes drift estimation in inertial navigation. As these variables are from far less observable than the body acceleration in the considered exemple, a substantial gain in estimation quality can be expected. As outlook of this work, it would be interesting to compare this method with others approaches using linear (and so different) inequality constraints, such as the probability density function truncation [11]. It would also be interesting to study the consequence of a first order linearization of the constraint equations on the proposed algorithm.

\section{REFERENCES}

[1] R.E. Kalman. "A new approach to linear filtering and prediction problems". Transactions of the ASME-Journal of Basic Engineering, Series D, 82:35-45, 1960.

[2] M.S. Grewal, L.R. Weill, and A.P. Andrews. "Global Positioning Systems, inertial navigation and intergration". Wiley \& sons inc., 2001.

[3] Y. Bar-Shalom. Multitarget multisensor tracking; Applications and advances, volume 3. Artech house, Norwood, Ma., 2001.

[4] B.D.O. Anderson and J.B. Moore. "Optimal filtering". Prentice Hall inc., 1979.

[5] B.D.O. Anderson and J.B. Moore. "Optimal control : linear quadratic methods". Prentice Hall inc., 1989.

[6] S. Simani, C. Fantuzzi, and R.J. Patton. Model-based fault diagnosis in dynamic systems using identification techniques. Springer Verlag inc., 2002.

[7] D. Simon and T. Chia. Kalman filtering with state equality constraints. IEEE transactions on Aerospace and Electronic Systems, 38(1):128136, 2002.

[8] D. Choukroun. Novel methods for attitude determination using vector observations. PhD thesis, Israel Institute of Technology, 2003.

[9] W. Wen and H. Durrant-Whyte. Model-based multi-sensor data fusion. In IEEE conference on Robotics and Automation, pages 1720-1726, Nice, France, 1992.

[10] S. Hayward. Constrained kalman filter for least squares estimation of time-varying beamforming weights. Mathematics in Signal Processing, IV:113-125, 1998.

[11] D. Simon. Optimal state estimation. Kalman, $H_{\infty}$ and nonlinear approaches. Wiley \& sons inc., 2006.

[12] D. Simon and D.L. Simon. Kalman filtering with inequality constraints for turbofan engine health estimation. IEE proceedings on Control Theory and Applications, 153(3):371-378, 2006.

[13] R. Fletcher. Practical methods for optimization. Volume 2: constrained optimization. Wiley \& sons inc., 1981.

[14] Y. Bar-Shalom and K. Birmiwal. "Variable dimension filter for maneuvering target tracking". IEEE transactions on Aerospace and Electronic Systems, 18(5):621-629, 1982.

[15] Y.T. Chan, A.G.C Hu, and J.B. Plant. "A Kalman filter based tracking scheme with input estimation". IEEE transactions on Aerospace and Electronic Systems, 15(2):237-244, 1979.

[16] T.C. Wang and P.K. Varshney. "A tracking algorithm for maneuvering targets". IEEE transactions on Aerospace and Electronic Systems, 29(3):910-924, 1993.

[17] H. Lee and M.J. Tark. Generalized input estimation technique for tracking maneuvering targets. IEEE transactions on Aerospace and Electronic Systems, 35(4):1388-1402, 1999.

[18] P.L. Bogler. "Tracking maneuvering targets using input estimation". IEEE transactions on Aerospace and Electronic Systems, 23(3):298310, 1987.

[19] H.A.P. Blom and Y. Bar-Shalom. "The Iteracting Multiple Model algorithm for systems with Markovian switching coefficients". IEEE transactions on Automatic Control, 33(8):780-783, 1988.

[20] A. Hocine, M. Chadli, D. Maquin, and J. Ragot. A discrete-time sliding window observer for markovian switching system. In 45th IEEE Conference on Decision and Control, San Diego, Ca., USA, 2006 . 